\title{
Wafer Level Characterisation of Microelectrodes for Electrochemical Sensing Applications
}

\author{
E.O. Blair*, L. Parga Basanta ${ }^{\dagger}$, I. Schmueser ${ }^{\ddagger}$, J.R.K. Marland*, A. Buchoux ${ }^{\S}$, A. Tsiamis ${ }^{\dagger}$ \\ C. Dunare*, M. Normand*, A.A. Stokes*, A.J. Walton* and S. Smith ${ }^{\dagger}$ \\ *School of Engineering, Institute for Integrated Micro and Nanosystems \\ ${ }^{\dagger}$ School of Engineering, Institute for Bioengineering \\ ${ }^{\ddagger}$ School of Chemistry \\ $\S$ School of Engineering, Institute for Multiscale Thermofluids \\ The University of Edinburgh, Alexander Crum Brown Road, \\ Edinburgh, EH9 3FF, UK \\ Email: Stewart.Smith@ed.ac.uk \\ Tel: +44 (131) 6507471
}

\begin{abstract}
This work presents a system for the in-line waferlevel characterisation of electrochemical sensors. Typically, such sensors are first diced and packaged before being electrochemically tested. By integrating their characterisation into the manufacturing process, the production of electrochemical sensors becomes more efficient and less expensive as they can be parametrically tested midway through the fabrication process, without the need to package them. This enables malfunctioning or failed devices to be identified before dicing and reduces costs as only functional devices are packaged (in many cases this can be more expensive than the sensor fabrication). This study describes wafer-level characterisation of a simple electrochemical sensor design using a photoresist hydrophobic corralling film for the electrolyte and a probe station for contacting to individual dies.
\end{abstract}

\section{INTRODUCTION}

Electrochemical sensors are a popular tool in a wide variety of applications, ranging from industrial monitoring to fundamental science [1]-[4]. One of the most common methods of manufacturing electrochemical sensors is using microfabrication, which allows for controlled and reproducible production of nominally identical sensors. Such sensors are also easily miniaturised to the micron scale and are compatible with CMOS processes [5]-[7]. This presents the opportunity to adapt metrology techniques typically used in semiconductor manufacturing, specifically wafer-level measurements of devices, for characterising electrochemical systems before packaging [8]. Typically, electrodes are characterised after dicing and packaging, which is an expensive and time-consuming element of sensor production. This work demonstrates a system for characterising electrochemical sensors at wafer level. The benefits of this include the ability to rapidly characterise many different electrode designs without the need to package devices. This will help expedite the development of electrode technologies through systematic assessment of adjustments to the electrode design, by monitoring electrochemical test structures. Additionally, this approach enables the identification of malfunctioning devices, as well as the verification and process control of microfabrication steps.

To demonstrate the validity of the proposed system, quantifiable electrochemical measurements are performed in a manual probe station using electrochemical test structures. These are compared with measurements made in a traditional electrochemical measurement setup. Following this, the capability of the probe station setup to spatially map electrochemical responses across the wafer is demonstrated. The requirements for a completely automatic system are also discussed.

\section{Test Structure Design And FAbricAtion}

\section{A. Test Structure Design}

The test structures consist of a three-electrode cell on a $3 \mathrm{~mm}$ square die, pictured in Fig. 1. The central working electrode (WE) is where the potential and current of interest are applied and measured. The inner ring electrode around the WE is a pseudo-reference electrode (RE), which provides a reference potential against which potentials at the WE are applied and measured. The outer ring electrode forms the counter electrode $(\mathrm{CE})$, which supplies the current required for the chemical reactions taking place at the WE. The WE designs in this work are single square and disc microelectrodes with a range of edge lengths/diameters $(20,50$, and $100 \mu \mathrm{m})$, along with Cartesian arrays of discs of various sizes and spacings (20 and $50 \mu \mathrm{m}$ diameter with pitches of 100 and 150 $\mu \mathrm{m})$. A layer of patterned positive photoresist was used as a corral to help contain the liquid over the electrodes, and this is visible as a square pattern around the electrodes in Fig. 1.

\section{B. Test Structure Fabrication}

The test structure design consisted of a metal layer sandwiched between two insulation layers. Firstly, a $500 \mathrm{~nm}$ thick thermal silicon dioxide $\left(\mathrm{SiO}_{2}\right)$ insulator was grown on a 100 $\mathrm{mm}$ silicon $(\mathrm{Si})$ substrate. $50 \mathrm{~nm}$ of platinum $(\mathrm{Pt})$ with a 


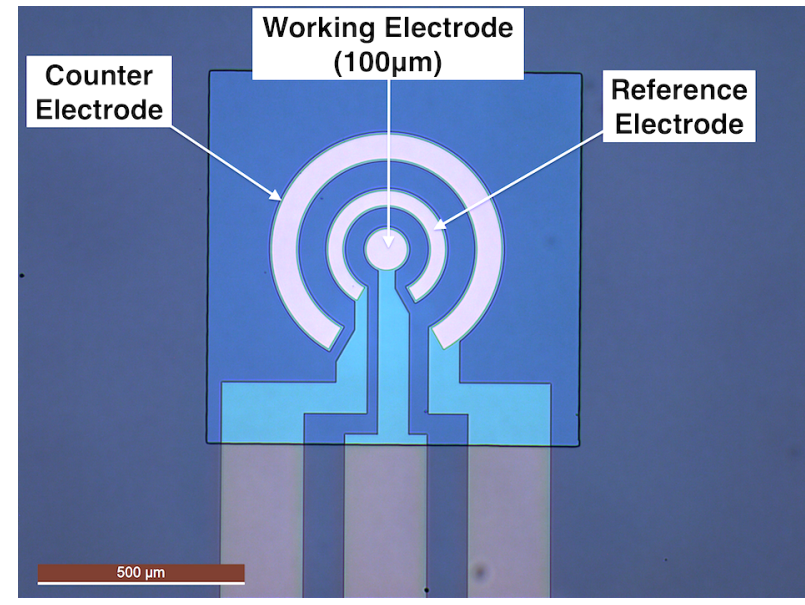

Fig. 1. Photograph of test structure layout, including the on-chip threeelectrode system.

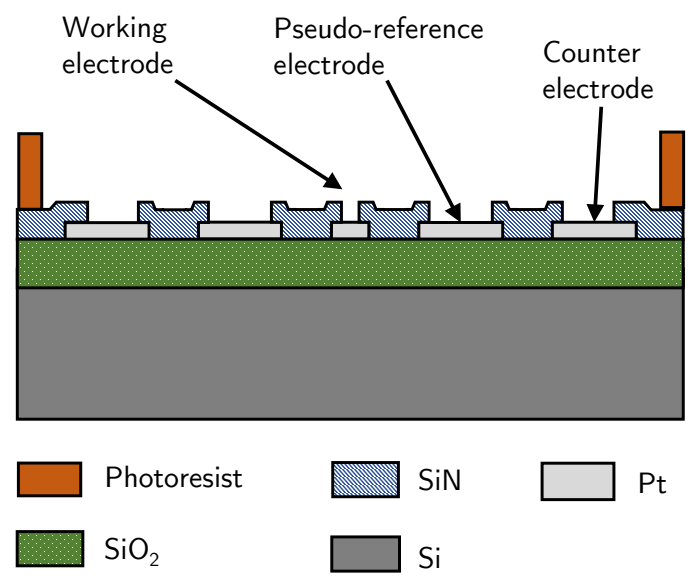

Fig. 2. A cross section of the test structure design showing the layers, with the electrodes labelled. The thickness of the layers have been exaggerated for clarity.

$10 \mathrm{~nm}$ titanium (Ti) adhesion layer was then deposited and patterned to create electrodes, contact pads, and interconnects. $500 \mathrm{~nm}$ of silicon nitride $(\mathrm{SiN})$ was then deposited using plasma enhanced chemical vapour deposition to insulate the metal from the solution. Windows were then etched into this insulation to provide access to the contact pads and electrodes. A cross section (left to right in Fig. 1) of the die through the WE is presented in Fig. 2.

\section{Hydrophobic Liquid Corral}

To help corral the liquid electrolyte over the electrodes, a layer of SPR 350-1.2 (Rohm and Haas) photoresist was spin coated onto the wafer and patterned to expose the contact pads (for electrical connections) and a $900 \mu \mathrm{m}$ edge length square area over the electrodes. As the photoresist is less hydrophilic than the SiN insulator, it helped prevent liquid wicking up the to the contact pads. To demonstrate this, Table I presents contact angle measurements of the photoresist
TABLE I

The AVERAGE MEASURED CONTACT ANGLES OF THE SiN, PT, AND Photoresist SURFACES, $N=6( \pm 3 \sigma)$

\begin{tabular}{|c||c|}
\hline Material & Contact Angle \\
\hline Silicon Nitride & $36^{\circ} \pm 7^{\circ}$ \\
SPR350-3 & $70^{\circ} \pm 8^{\circ}$ \\
\hline
\end{tabular}

and SiN made using de-ionised (DI) water. The difference in mean contact angle of $34^{\circ}$ is sufficient to help confine the liquid to the electrode area. Fig. 3(a) and (b) show a photograph of the liquid electrolyte contained within the more hydrophilic pattern in the photoresist. If a larger difference in hydrophobicity is desired, more hydrophobic materials such as polytetrafluoroethylene (PTFE) or Parylene- $\mathrm{C}^{\mathrm{TM}}$ could be employed [9], [10]. However, this requires considerably more processing, which is not desirable in the middle of a preexisting process. Although photoresist is not hydrophobic, its benefit lies in its simplicity to apply and then remove once testing is finished.

\section{EXPERIMENTAL METHODS}

\section{A. Experimental Setup}

The electrochemical measurements were made using an Everbeing EB8 manual probe station inside a shielded dark box. The probe needles were placed on the die contact pads and connected to an Autolab Micro III potentiostat (Metrohm). The solution was dispensed through a micro-pipette tip which was attached to another micromanipulator arm and placed over the electrode area. Silicone tubing connected the pipette tip to a syringe, enabling control over the flow of liquid. The full experimental set up on the manual probe station is presented in Fig.4.

Results from this setup were compared against benchmark measurements made in a traditional electrochemical setup. This consisted of a glass beaker in a Faraday cage, with separate Pt CE and pseudo RE. These electrodes were shards of $\mathrm{Si}$, coated in $500 \mathrm{~nm} \mathrm{SiN}$ and then $50 \mathrm{~nm}$ of Pt on a 10 $\mathrm{nm}$ Ti adhesion layer. The WE was a $20 \mu \mathrm{m}$ diameter Pt disc.

\section{B. Measurements}

A standard electrochemical measurement technique called cyclic voltammetry was used to characterise the electrodes in the probe station setup. This involves sweeping the potential at the WE (vs. the RE) between two defined values and back while monitoring the current. This measurement is typically performed in an electrolyte containing a substance which undergoes electrochemical reduction and oxidation in the chosen potential range, a redox couple. Ferri/ferrocyanide $\left(\left[\mathrm{Fe}(\mathrm{CN})_{6}\right]^{3-} /\left[\mathrm{Fe}(\mathrm{CN})_{6}\right]^{4-}\right)$ is a commonly used and wellunderstood redox couple [11], [12], and was hence used for the experiments in this paper. The solution used throughout this work was $1 \mathrm{mM}$ of both potassium ferricyanide and potassium ferrocyanide in a background electrolyte of $100 \mathrm{mM}$ potassium chloride. 


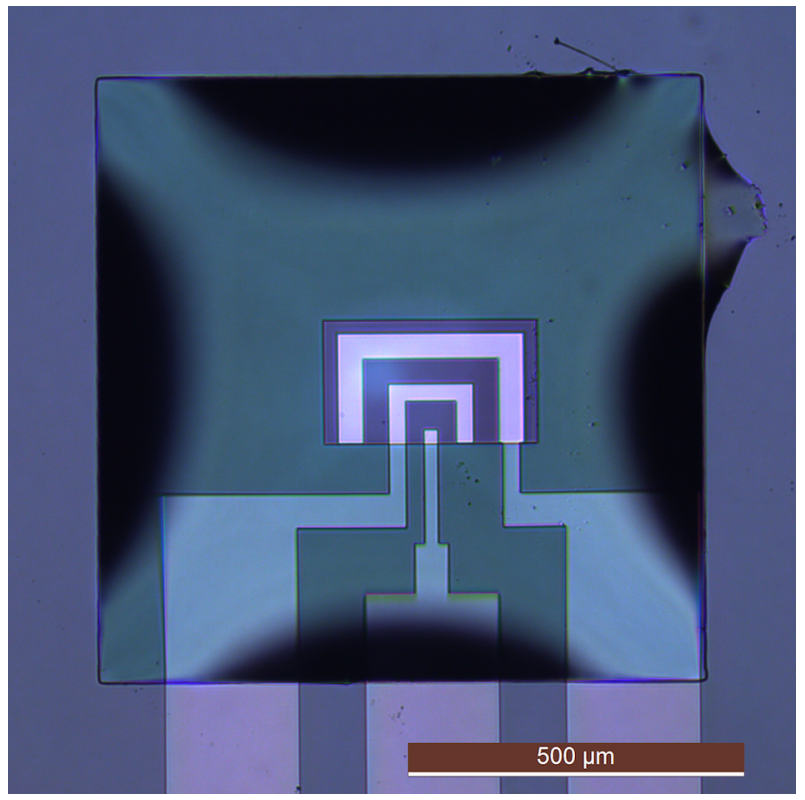

(a)

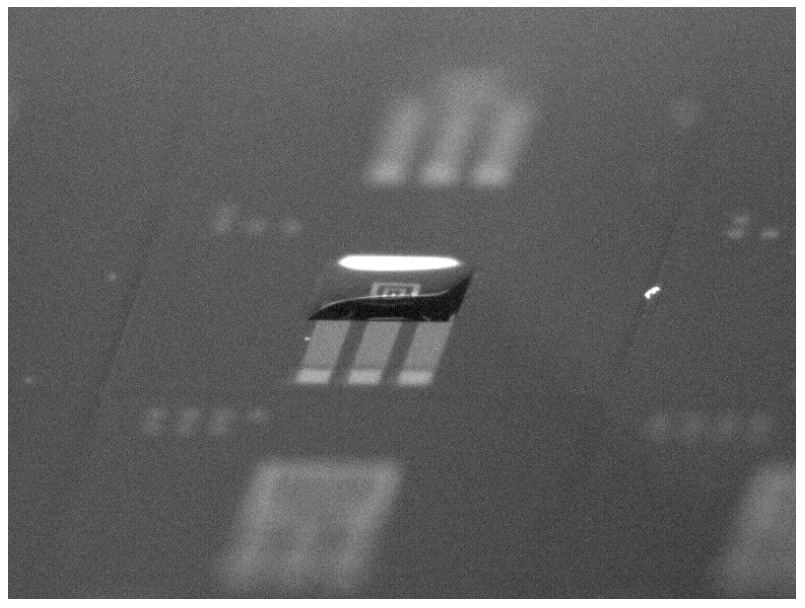

(b)

Fig. 3. Photographs showing the photoresist corralling DI water over an electrode (a) from the top down and (b) at an angle.

Despite the shielding of the probe station, it was observed that the measurements recorded on the probe station setup suffered from electrical noise superimposed on the electrochemical response. Therefore, a Fast Fourier Transform smoothing filter over an 8 point window was used to filter the data using Origin 2016 graphing and analysis software (Originlab).

\section{Measurement Results}

\section{A. Comparison of On-Wafer and In-Beaker Results}

Firstly, the capability of making electrochemical measurements at wafer level was assessed by comparing those made using in the probe station set up, with those made in a traditional electrochemical system. A cyclic voltammogram (CV) of the redox reaction of ferri/ferrocyanide recorded in (a) a beaker and (b) the probe station setup is presented in Fig. 5.

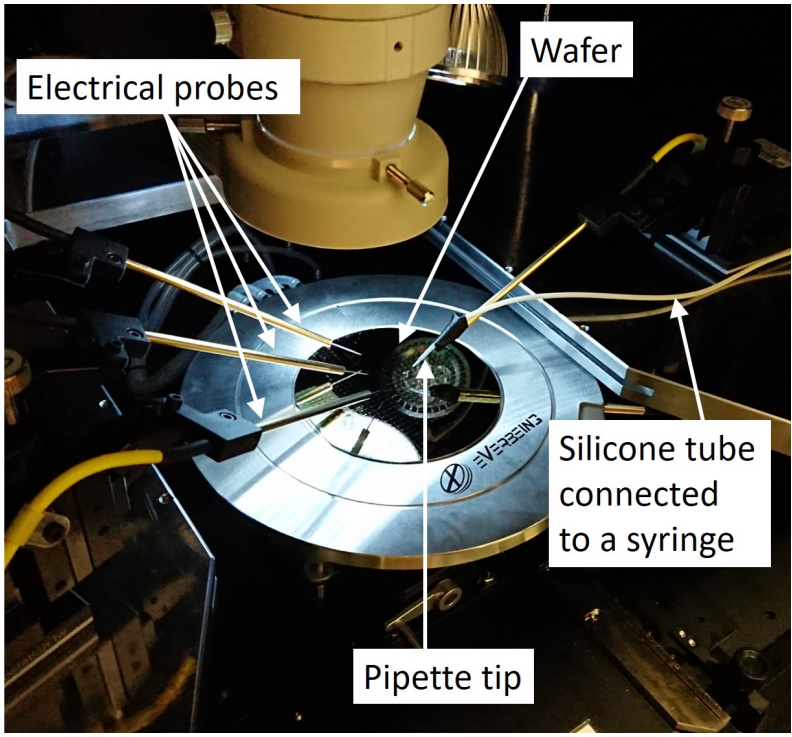

Fig. 4. Photographs of the experimental set up showing the probe station, electrical probes, and pipette tip.

TABLE II

THE AVERAGE MEASURED LIMITING CURRENT OF THE TWO SETUPS, $N=5( \pm 3 \sigma)$, COMPARED AGAINST THE PREDICTED VALUE.

\begin{tabular}{|c||c|}
\hline Experiment & Current (nA) \\
\hline Predicted & 2.57 \\
Wafer Level & $2.77 \pm 0.45$ \\
Beaker & $2.48 \pm 0.29$ \\
\hline
\end{tabular}

The shape of the CV is the expected wave in both set ups, characteristic of the electrochemical response of a microelectrode. As the potential is swept positive, ferrocyanide is oxidised to ferricyanide generating a positive current, while during the negative potential sweep, a negative current is generated by the reduction of ferricyanide to ferrocyanide. Under the presented conditions, the current is mass transport controlled by the diffusion of analyte to the electrode and reaches a steady state current $\left(i_{L}\right)$. This current can be quantified using [13], [14],

$$
i_{L}=B n F D c r
$$

where $n$ is the number of electrons transferred in the reaction, $F$ is the Faraday constant, $D$ and $c$ are the diffusion coefficient and concentration of the reactant respectively, and $r$ is the radius of a disc electrode (or $l$, the edge length for a square). The $B$ term is a dimensionless coefficient dependant on the geometry of the electrode, and is equal to 4 for an inlaid disc and 2.3 for an inlaid square. This enabled the comparison of predicted and measured currents. Using a literature value for $D$ of $6.67 \times 10^{-10} \mathrm{~m}^{2} \mathrm{~s}^{-1}$ [15], the predicted limiting current for the reduction reaction was calculated and is presented in Table II alongside the measured currents from both systems.

The three values match each other within error, lending further confidence that the probe station set up can be used to record quantifiable electrochemistry. Furthermore, the wave 


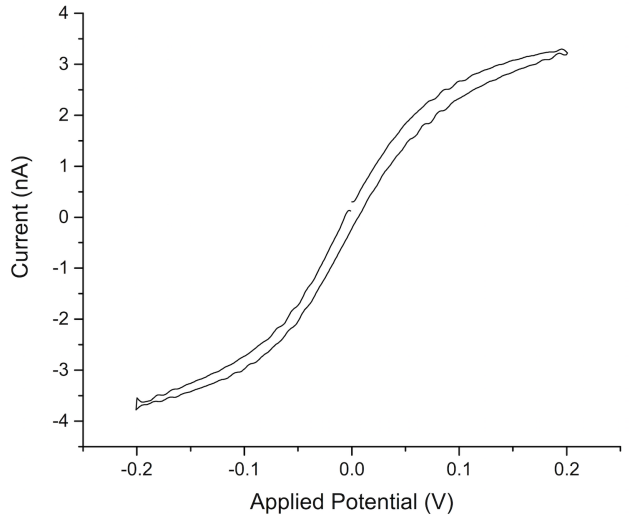

(a)

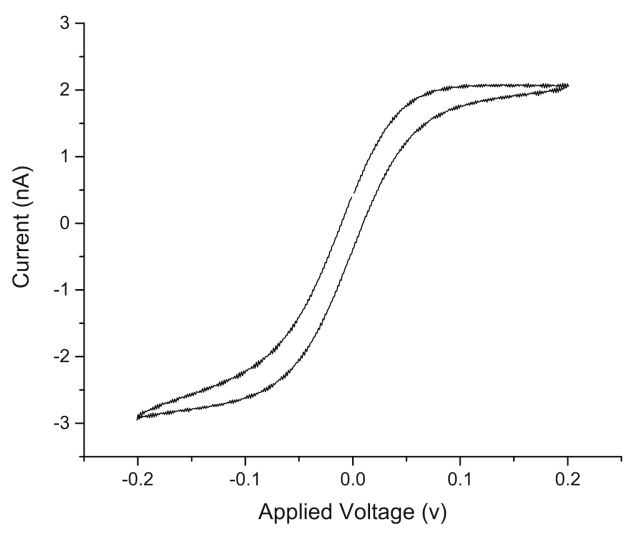

(b)

Fig. 5. (a) $\mathrm{CV}$ recorded on a $20 \mu \mathrm{m}$ diameter disc electrode at $100 \mathrm{mVs}^{-1}$ in the probe station and (b) the same measurement in the beaker setup.

shape of the CVs are typical of an electrochemical reaction happening at a micro-scale electrode (where diffusion is the rate limiting step).

It is noteworthy that the variation in the wafer-level value is higher than the beaker. This could be due to the noisier measurements from the probe station, potentially arising from the leads to the potentiostat, which was not in the shielded box around the probe station. Another source of variation is the difference in ambient temperature between the two setups or, more likely, changes in concentration of the redox couple over time. This was seemingly caused by evaporation of water from the droplet, resulting in the relative concentration of redox couple increasing. This effect can be seen in the CVs presented in Fig. 6, where the currents associated with the reduction and oxidation reactions increase over time. Eventually the current suddenly decreases to near zero indicating complete evaporation of the liquid and thus loss of electrical contact between the electrodes. Another difference between the two setups is the difficulty in electrochemically cleaning electrodes on the wafer, arising from the requirement of changing be-

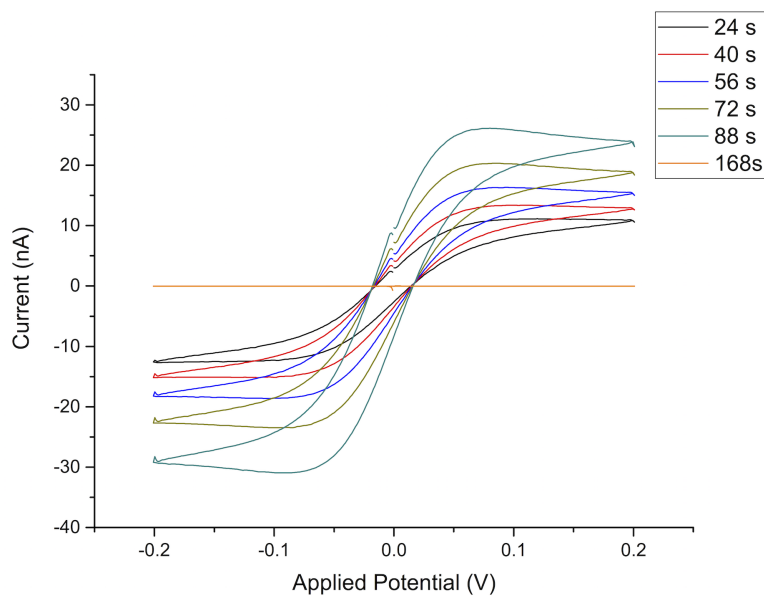

Fig. 6. CVs recorded over time on a $50 \mu \mathrm{m}$ diameter disc electrode at 100 $\mathrm{mVs}^{-1}$.

TABLE III

DETAILS OF ELECTRODE DESIGNS.

\begin{tabular}{|c||c|c|c|}
\hline $\begin{array}{c}\text { Electrode } \\
\text { (Fig. 7(b)) }\end{array}$ & Geometry & Size $(\boldsymbol{\mu m})$ & $\begin{array}{c}\text { Pitch }(\boldsymbol{\mu m}) \\
\text { /no. of ectrodes }\end{array}$ \\
\hline (i) & Disc Array & 20 & $100 / 12$ \\
(ii) & Disc Array & 50 & $100 / 9$ \\
(iii) & Disc Array & 50 & $150 / 9$ \\
(iv) & Disc & 20 & $-/ 1$ \\
(v) & Disc & 50 & $-/ 1$ \\
(vi) & Disc & 100 & $-/ 1$ \\
(vii) & Square & 20 & $-/ 1$ \\
(viii) & Square & 50 & $-/ 1$ \\
(ix) & Square & 100 & $-/ 1$ \\
\hline
\end{tabular}

tween a cleaning solution and one containing the analyte for measuring. As a consequence, this likely contributed to the slight differences in shape between the CVs as well as the increased error for the probe station measurement.

\section{B. Spatial Electrode Measurements}

Wafer-level mapping of devices can be a powerful tool for understanding fabrication processes, as well as quantifying parameters such as yield and uniformity [16]. To demonstrate that capability can be expanded to electrochemical sensors, the same cyclic voltammetry measurements were performed across nine neighbouring electrodes shown in Fig. 7(a). The recorded CVs are shown in Fig. 7(b), and Table III lists the details of the designs of the electrodes. All the electrodes gave the expected electrochemical response, with the larger $100 \mu \mathrm{m}$ electrodes shown in Fig. 7(b) (vi) and (ix) showing peaks characteristic of electrochemical reactions occurring at the surface of larger, macro-scale electrodes. This transition between macro- and microelectrode behaviour typically occurs between sizes of $2050 \mu \mathrm{m}$ and can be observed in Fig. 7(b) (v) and (vi). The arrays (Fig. 7(b) (i), (ii) and (iii)) show larger currents, proportional to the number of electrodes, while retaining the wave-like $\mathrm{CV}$ response. 


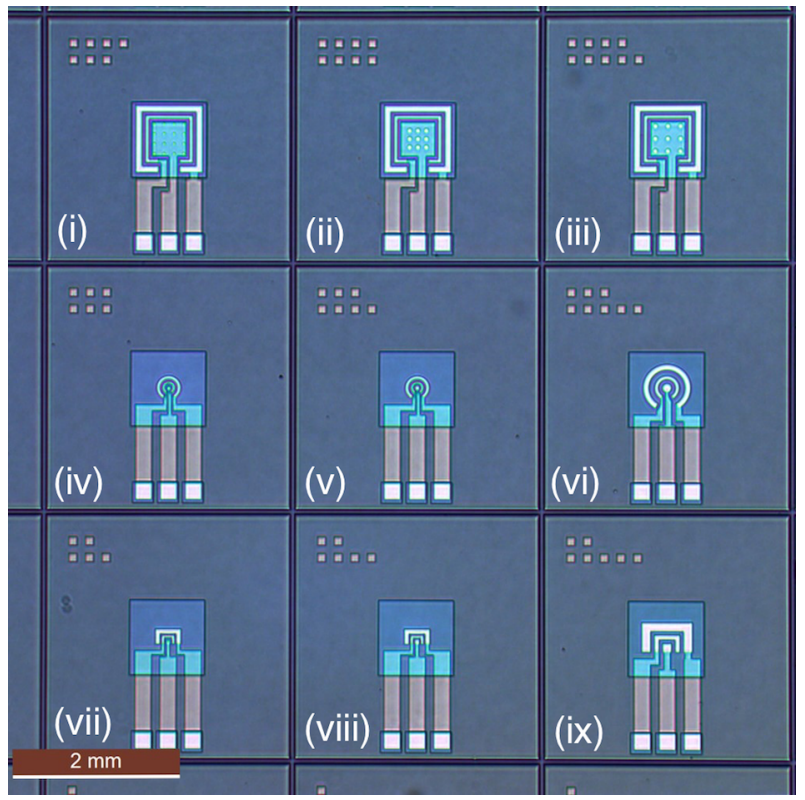

(a)
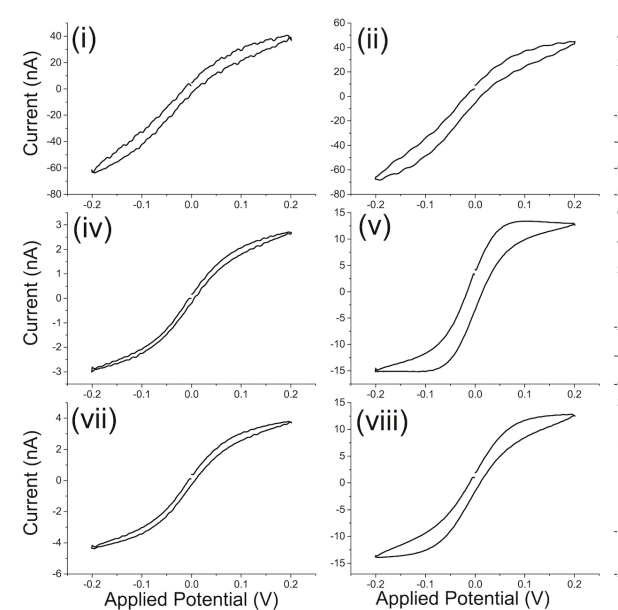

(b)

Fig. 7. (a) The nine electrodes on the wafer surface which were used to record the (b) CVs.

\section{Discussion}

The results in Table II and Fig. 5 confirm that quantifiable electrochemical measurements can be made at wafer level using a standard probe station setup. However, the evaporation of the droplet presents a complication, as it both imposes a maximum measurement time and a change in analyte concentration over time. This makes quantification of the current, which typically requires a known concentration, beyond 30 to 60 seconds challenging. This also limited the time of the experiment as the droplets, which were typically around $1 \mathrm{~mm}$ across, evaporated completely in under 5 minutes in ambient conditions. While this presents a challenge for some measurements, it enables others, such as electrochemical monitoring of droplet evaporation. There are also methods of removing the evaporation factor. For example, a solution could be to employ a seal such as that used by [17]. which would prevent the liquid evaporating or to have a constant new supply of solution to compensate. Although salt residue is left on the surface of the wafer once the droplet is dried, this is easily washed off with DI water. Electrochemical cleaning also presents a difficulty as changing between cleaning and measurement solutions would expose the electrode air, allowing reformation of surface oxides. This could be resolved by combining the redox and cleaning solution, although this is not possible in all cases.

Even with evaporation, the set up can be used to confirm operation of electrochemical sensors and identify faulty devices, as the value of limiting current is dependent on radius (or edge length of a square) through equation 1. Should the current deviate from the predicted value, then this could be indicative of pinholes or other defects in the top insulator. This process is much faster than making measurements in a beaker setup, especially in the case miniaturised sensors like those presented in this paper, which typically require packaging to insulate nearby wire bonds and contact pads. Being able to perform wafer-level electrochemical testing enables the fast collection of a large number of measurements. For example, setting up and then recording $10 \mathrm{CVs}$ at each of the nine different electrodes presented in Fig. 7(b) took around 10 minutes. This process could be further expedited with the incorporation of both automatic liquid dispenser and wafer stage, enabling a fully automatic electrochemical measurement setup.

Future work is to develop the set up along the lines described above. An example of this is the bespoke microfluidic system, under development to replace the pipette tip shown in Fig. 8. The component is made from PDMS and was manufactured using a 3D printed scaffold made of Acrylonitrile Butadiene Styrene. PDMS was then cast around this and the scaffold dissolved in acetone [18]. It consists of two inlets at the top, one for solution and one for a non-pseudo reference electrode which will expand the range of possible electrochemical measurements. The outlet at the bottom is designed to seal around the electrode area, mitigating evaporation of the solution.

\section{CONCLUSION}

A method of making electrochemical measurements at wafer-level has been demonstrated. This consists of a fluidic system for dispensing droplets in a standard probe station and was validated with wafer-level electrochemical test structures. Electrochemical measurements were performed on a wafer and, when quantified, matched the performance of those made in a conventional electrochemical cell. Electrodes of different designs were then measured and gave the expected response, demonstrating the rapid and simple characterisation of multiple electrode designs across a wafer.

\section{ACKNOWLEDGMENT}

This work was funded by the UK Engineering and Physical Sciences Research Council, through the IMPACT programme grant $(\mathrm{EP} / \mathrm{K} 034510 / 1)$ and the PACIFIC programme grant 


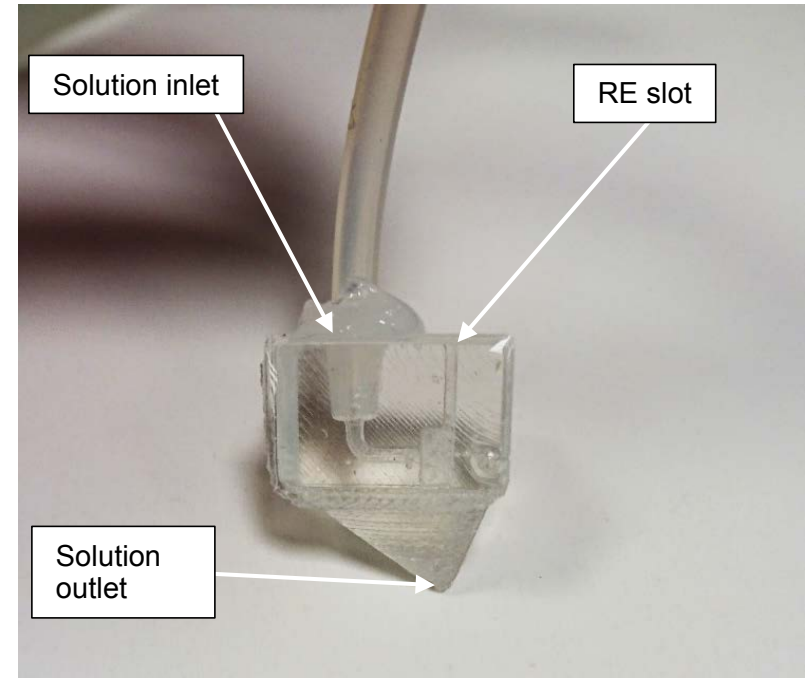

Fig. 8. Photograph of a microfluidic component, designed to replace the pipette tip.

(EP/L018616/1). We would also like to acknowledge the support of the EPSRC CDT in Intelligent Sensing and Measurement. All data presented in this paper can be accessed at http://dx.doi.org/10.7488/ds/2295.

The authors would like to acknowledge the support of Everbeing for the donation of the manual probe station used in this work.

\section{REFERENCES}

[1] D. K. Corrigan, J. P. Elliott, E. O. Blair, S. J. Reeves, I. Schmüser, A. J. Walton, and A. R. Mount, "Advances in electroanalysis, sensing and monitoring in molten salts," Faraday Discussions, vol. 190, pp. 351366, 2016.

[2] E. González-Fernández, N. Avlonitis, A. F. Murray, A. R. Mount, and M. Bradley, "Methylene blue not ferrocene: Optimal reporters for electrochemical detection of protease activity," Biosensors and Bioelectronics, vol. 84, pp. 82-88, Oct. 2016.

[3] M. A. Johnson, "In vivo electrochemical measurements: past, present and future," Bioanalysis, pp. 119-122, 2013.

[4] R. J. Forster, "Microelectrodes: new dimensions in electrochemistry," Chemical Society Reviews, vol. 23, no. 4, pp. 289-297, 1994.

[5] J. G. Terry, I. Schmüser, I. Underwood, D. K. Corrigan, N. J. Freeman, A. S. Bunting, A. R. Mount, and A. J. Walton, "Nanoscale electrode arrays produced with microscale lithographic techniques for use in biomedical sensing applications," IET nanobiotechnology, vol. 7, no. 4, pp. 125-134, Dec. 2013.
[6] N. Jamil, S. Smith, Y. Yang, J. Jia, P. Bagnaninchi, and E. GonzálezFernández, "Design and fabrication of microelectrodes for electrical impedance tomography of cell spheroids," in IECBES 2016 - IEEEEMBS Conference on Biomedical Engineering and Sciences, Feb. 2017, pp. $426-431$.

[7] E. O. Blair, D. K. Corrigan, J. G. Terry, A. R. Mount, and A. J. Walton, "Development and Optimization of Durable Microelectrodes for Quantitative Electroanalysis in Molten Salt," Journal Of Microelectromechanical Systems, vol. 24, no. 5, pp. 1346-1354, 2015.

[8] S. Bahukudumbi and K. Chakrabarty, Wafer-Level Testing and Test During Burn-In for Integrated Circuits. Artech House, 2010.

[9] H. Zou, Y. Li, S. Smith, A. Bunting, A. J. Walton, and J. Terry, "Modification and characterisation of material hydrophobicity for surface acoustic wave driven microfluidics," in Microelectronic Test Structures (ICMTS), 2012 IEEE International Conference on, 2012, pp. 61-65.

[10] Y. Li, E. O. McKenna, W. Parkes, P. A. R., and A. J. Walton, "The application of fixed hydrophobic patterns for confinement of aqueous solutions in proteomic microarrays," Appl. Phys. Lett., vol. 99, pp. 073 703-1 - 073 703-3, 2011.

[11] P. H. Daum and C. G. Enke, "Electrochemical kinetics of the ferriferrocyanide couple on platinum," Analytical Chemistry, vol. 41, no. 4, pp. 653-656, May 2002.

[12] J. J. Van Benschoten, J. Y. Lewis, W. R. Heinemann, D. A. Roston, and P. T. Kissinger, "Cyclic voltammetry experiment," Journal of CHemical Education, vol. 60, no. 9, pp. 772 - 776, 1983.

[13] Y. Saito, "A theoretical study on the diffusion current at the stationary electrodes of circular and narrow band types," Review of Polarography (Japan), vol. 15, no. 6, pp. 177 - 187, 1968.

[14] I. Schmueser, A. J. Walton, J. G. Terry, H. L. Woodvine, N. Freeman, and A. R. Mount, "A systematic study of the influence of nanoelectrode dimensions on electrode performance and the implications for electroanalysis and sensing," Faraday Discussions, vol. 164, pp. 295 - 314 2013.

[15] S. J. Konopka and B. McDuffle, "Diffusion coefficients of ferri- and ferrocyanide ions in aqueous media, using twin-electrode thin-layer electrochemistry," Analytical Chemistry, vol. 42, no. 14, pp. 1741 1746, 1970.

[16] S.-C. Hsu and C.-F. Chien, "Hybrid data mining approach for pattern extraction from wafer bin map to improve yield in semiconductor manufacturing," International Journal of Production Economics, vol. 107, pp. $88-103,2007$.

[17] A. Poghossian, K. Schumacher, J. Kloock, C. Rosenkranz, J. Schultze, M. Müller-Veggian, and M. Schöning, "Functional Testing and Characterisation of ISFETs on Wafer Level by Means of a Micro-droplet Cell," Sensors, vol. 6, no. 4, pp. 397-404, Apr. 2006.

[18] A. Buchoux, P. Valluri, S. Smith, A. A. Stokes, P. R. Hoskins, and V. Sboros, "Manufacturing of microcirculation phantoms using rapid prototyping technologies," 2015 37th Annual International Conference of the IEEE Engineering in Medicine and Biology Society (EMBC), pp. 5908-5911, Aug. 2015 